\title{
A Numerical Analyst's Fifteen-Foot Shelf
}

Imagine a laboratory of numerical analysis, with computers, coders, problem analysts, and research mathematicians. The group varies in mathematical experience from new college graduates to professionals with long research records. Disappointingly few can profitably consult a book not in English but, pooling talents, the group can read English, French, German, Italian, Russian, and Hebrew quite well. Quite as important as a comprehensive library (assumed to lie within a few miles) is a small library in the laboratory building. What should such a working library contain?

Clearly the library needs a diversity of material, of which at least five classes can be distinguished:

A. Mathematics books.

B. Books on computing machines.

C. Tables of functions.

D. Periodicals.

E. General references (e.g., language dictionaries).

This article is a proposed list of about 150 essential titles in class A. The reader is warned that the list has been hastily prepared and is very tentative; its inclusions and omissions should not be taken too seriously.

Class A was divided into the following five categories, of which four have been split into subcategories:

1. Bibliographies on mathematics.

2. Collections of formulas.

3. Books on numerical analysis.

4. Other books on applied mathematics.

5. Books on pure mathematics.

In selecting the titles five qualities were explicitly considered: (a) adequacy of material in topics likely to be needed; (b) use of English language; (c) completeness of bibliography; (d) readability; (e) recency. The order of precedence given to these qualities depended on the book user; for mature research men it was perhaps a, c, e, b, d, (most important first), while for junior computers, perhaps b, d, a, e, c. In categories 1, 2, and 3 a considerable proportion of the available books has been listed, so that any enlargement of the library would occur mainly in categories 4 and 5.

The bibliographical citations came from the books themselves, from the Library of Congress cards, or from Parke's very helpful Guide (see just below). Mrs. Mildred MarTinolich helped prepare the citations.

\section{BIBLIOGRAPHIES}

Engineering Research Associates, High-speed Computing Devices. McGraw-Hill, New York, 1950. $451 \mathrm{p}$.

A. Fletcher, J. C. P. Miller, \& L. Rosenhead, An Index of Mathematical Tables. McGraw-Hill, New York, 1946. 450 p.

A. D. Franki In \& E. V. HankaM, Bibliography on the Use of IBM Machines in Science, Statistics, and Education. International Business Machines Corp., New York, 1952. 50 p. 
L. E. Grinter, editor, Numerical Methods of Analysis in Engineering. Macmillan, New York, 1949. 207 p. [The article by T. J. HigGins contains a comprehensive bibliography on numerical solution of partial differential equations.]

HaRvard University, Staff of the Computation Laboratory, A Manual of Operation for the Automatic Sequence Controlled Calculator. Harvard University Press, 1946. 561 p.

A. G. Kurosh, A. I. Markushevich, \& P. K. Rashevskif, editors, Matematika v SSSR $z a$ Tridtsat' Let 1917-1947 [Mathematics in the U.S. S. R. in the Thirty Years 1917-1947]. Moscow-Leningrad, 1948. 1044 p. [Surveys and bibliographies of most Soviet work in this period.]

N. G. PARke III, Guide to the Literature of Mathematics and Physics. McGraw-Hill, New York, 1947. 205 p.

J. A. Shohat, E. Hille, \& J. L. Walsh, A Bibliography on Orthogonal Polynomials. National Research Council of the National Academy of Sciences, Washington, 1940. 204 p.

V. Z. Vlasov, V. V. Golubev, \& N. D. MoǏseEv, editors, Mekhanika v SSSR za Tridtsat' Let 1917-1947 [Mechanics in the U.S. S. R. in the Thirty Years 1917-1947] (Russian). Moscow-Leningrad, 1950. 416 p. [Companion volume to that of KUROSH and others.]

\section{COLLECTIONS OF FORMULAS}

\section{a. General}

E. P. AdAMS \& R. L. Hippisley, Smithsonian Mathematical Formulae and Tables of Elliptic Functions. Smithsonian Institution, Washington, 1947. $314 \mathrm{p}$.

D. Bierens de HaAn, Nouvelles Tables d'Intégrales Définies. Corrected edition, Stechert, New York, 1939. 716 p.

O. T. BÜRKLEN \& F. RingleB, Mathematische Formelsammlung. 3rd edit., W. de Gruyter, Berlin-Leipzig, 1936. 272 p.

H. B. Dwight, Tables of Integrals and other Mathematical Data. Macmillan, 1947. 250 p.

G. DoETsCH, Tabellen zur Laplace-Transformation und Anleitung zum Gebrauch. Springer, Berlin-Göttingen, 1947. 185 p.

W. GRÖBNER \& N. HOFREITER, Integraltafel. Erster Teil, Unbestimmte Integrale. Springer, Vienna-Innsbruck, 1949. 166 p.

A. HALD, Statistical Tables and Formulas. Wiley, New York, 1952. 97 p.

Cecil Hastings Jr., Approximations in Numerical Analysis. Looseleaf sheets being published by the RAND Corporation, Santa Monica, California, $1950 \mathrm{ff}$. 74 sheets so far.

L. B. W. Jolley, Summation of Series. Chapman and Hall, London, 1925. 232 p.

E. Madelung, Die mathematischen Hilfsmittel des Physikers. 3rd edition, American reprint, Dover, New York, 1943. 384 p.

W. MAGNUS \& F. Oberhetringer (translated by J. Wermer), Formulas and Theorems for the Special Functions of Mathematical Physics. Chelsea, New York, 1949. 172 p.

W. MEYER ZUR CAPELLEN, Integraltafeln. Sammlung unbestimmter Integrale elementarer Funktionen. Springer, Berlin-Göttingen-Heidelberg. 1950. $292 \mathrm{p}$.

Nautical Almanac Office (Great Britain), Interpolation and Allied Tables. H. M. Stationery Office, London, 1936. 45 p.

G. PAPELIER, Formulaire de Mathématiques Spéciales. 11th edition, Librairie Vuibert, Paris, 1947. 208 p. [Formulas from algebra, analysis, trigonometry, analytical geometry.]

\section{b. Bound with numerical tables}

L. J. Comrie, Chambers's Shorter Six-figure Mathematical Tables. Chambers, EdinburghLondon, 1950. $387 \mathrm{p}$.

H. T. DAvis, Tables of the Higher Mathematical Functions. Principia Press, Bloomington, Indiana, v. 1, 1933, 377 p.; v. 2, 1935, 391 p.

E. JAHNKE \& F. EMdE, Tables of Functions with Formulae and Curves. 4th edition, Dover, New York, 1945. $306+76$ p. 
National Bureau of Standards, Tables of Chebyshev Polynomials $C_{n}(x)$ and $S_{n}(x)$. Applied Mathematics Series, v. 9, Government Printing Office, Washington, 1952. 161 p. [The introduction by C. LANCzos is a valuable reference.]

\section{REFERENCES ON NUMERICAL METHODS}

\section{a. General}

D. R. Hartree, Numerical Analysis. Oxford University Press, 1952. 287 p.

A. S. Housenolder, Numerical Analysis. To be published by McGraw-Hill, New York, autumn 1953.

W. E. Milne, Numerical Calculus. Princeton University Press, 1949. 393 p. $371 \mathrm{p}$.

C. RUNGE \& H. KöNIG, Vorlesungen über numerisches Rechnen. Springer, Berlin, 1924.

J. B. Scarborough, Numerical Mathematical Analysis. 2nd edition, John Hopkins Press, Baltimore, 1950. 511 p.

H. von Sanden, Praktische Analysis. 2nd edition, Teubner, Leipzig, 1923. 195 p.

E. T. WhitTaker \& G. RoBinson, The Calculus of Observations. 4th edition, Blackie, London-Glasgow, 1944. 397 p.

F. A. Willers (translated by R. T. Beyer), Practical Analysis. Dover, New York, 1948. $422 \mathrm{p}$.

\section{b. Matrix problems}

P. S. Dwyer, Linear Computations. Wiley, New York, 1951. 344 p.

V. N. FADDEEVA, Vychislitel'nye Metody Lineinnoi Algebry [Computational Methods of Linear Algebra], Moscow-Leningrad, 1950. 240 p.

R. A. Frazer, W. J. Duncan, \& A. R. Collar, Elementary Matrices, and Some Applications to Dynamics and Differential Equations. Cambridge Univ. Press, 1938. 416 p.

National BuReau of STANDARDs, Simultaneous Linear Equations and the Determination of Eigenvalues. Applied Math. Series, v. 29, Government Printing Office.

R. ZURMÜHL, Matrizen. Springer, Berlin-Göttingen-Heidelberg, 1950. 427 p. [A very useful book.]

\section{c. Differential equations}

A. A. Bennett, W. E. Milne, \& H. Bateman, Numerical Integration of Differential Equations. National Research Council of the National Academy of Sciences, Washington, 1933. 108 p. [Extensive bibliographies.]

L. Collatz, Eigenwertaufgaben mit technischen Anwendungen. Akademische Verlag., Leipzig, 1949. 466 p.

L. Collatz, Numerische Behandlung von Differentialgleichungen. Springer, BerlinGöttingen-Heidelberg, 1951. 458 p.

L. V. Kantorovich \& V. I. Krylov, Priblizhennye Metody Vysshego Analiza [Approximate Methods of Higher Analysis]. 3rd edition, Moscow-Leningrad, 1950. 697 p.

W. E. Milne, Numerical Solution of Differential Equations. Wiley, New York, 1953. 275 p. [Extensive bibliography.]

R. V. Southwell, Relaxation Methods in Engineering Science. Oxford University Press, 1940. 252 p.

R. V. Southwell, Relaxation Methods in Theoretical Physics. Clarendon Press, Oxford, 1946. 248 p.

\section{d. "Monte Carlo" methods}

National Bureau of Standards, Monte Carlo Methods. Applied Mathematics Series, v. 12, U. S. Government Printing Office, Washington, 1951. 42 p. 


\section{REFERENCES ON APPLIED MATHEMATICS}

\section{a. Engineering mathematics}

A. Bronwell, Advanced Mathematics in Physics and Engineering. McGraw-Hill, New York, 1953. $475 \mathrm{p}$.

R. V. ChURChILL, Fourier Series and Boundary Value Problems. McGraw-Hill, New York, 1941. 206 p.

R. V. ChURCHILl, Modern Operational Mathematics in Engineering. McGraw-Hill, New York, 1944. $306 \mathrm{p}$.

R. E. DoherTy \& E. G. Keller, Mathematics of Modern Engineering. Wiley, New York, v. 1, 1936, 314 p.; v. 2 (by Keller only), 1942, 309 p.

M. F. Gardner \& J. L. Barnes, Transients in Linear Systems. v. 1, Wiley, New York, 1942. 389 p.

H. Margenau \& G. M. Murphy, The Mathematics of Physics and Chemistry. van Nostrand, New York, 1943. 581 p. $426 \mathrm{p}$.

R. Oldenburger, Mathematical Engineering Analysis. Macmillan, New York, 1950.

I. S. Sokolnikoff \& E. S. Sokolnikoff, Higher Mathematics for Engineers and Physicists. 2nd edition, McGraw-Hill, New York, 1941. 587 p.

\section{b. Theoretical physics} $864 \mathrm{p}$.

J. M. Blatt \& V. F. Weisskopf, Theoretical Nuclear Physics. Wiley, New York, 1952.

M. BoRN, Optik. Ein Lehrbuch der elektromagnetischen Lichttheorie. Reprint, Edwards, Ann Arbor, 1943. 591 p.

H. Jefrreys \& B. S. Jeffreys, Methods of Mathematical Physics. 2nd edition, Cambridge University Press, 1950.708 p. $748 \mathrm{p}$.

G. Joos (translated by I. M. Freeman), Theoretical Physics. Hafner, New York, 1934.

P. M. MoRse \& H. Feshbach, Methods of Theoretical Physics. (multigraphed) Technology Press, Cambridge, 1946. 497 p. [Being published by McGraw-Hill.]

L. I. Schiff, Quantum Mechanics. McGraw-Hill, New York, 1949. 404 p.

W. R. Smythe, Static and Dynamic Electricity. McGraw-Hill, New York, 1939. 560 p.

J. A. Stratton, Electromagnetic Theory. McGraw-Hill, New York, 1941. 615 p.

M. W. Zemansky, Heat and Thermodynamics. 2d edition, McGraw-Hill, New York, $1943,390 \mathrm{p}$.

\section{c. Mechanics}

H. Goldstein, Classical Mechanics. Addison-Wesley, Cambridge, 1950. 399 p.

E. J. McShane, J. L. Kelley, \& F. V. Reno, Exterior Ballistics. University of Denver Press, 1953. $834 \mathrm{p}$.

L. M. Milne-Thomson, Theoretical Hydrodynamics. 2d edition, Macmillan, London, 1949. $600 \mathrm{p}$.

LoRd Rayleigh (J. W. StRutT), The Theory of Sound. 2d edition, 2 vols. reprinted in one, Dover, New York, 1945. $480+504$ p.

R. H. Scanlan \& R. Rosenbaum, Introduction to the Study of Aircraft Vibration and Flutter. Macmillan, New York, 1951. 428 p.

E. T. Whittaker, A Treatise on the Analytical Dynamics of Particles and Rigid Bodies. 4th edition, Dover, New York, 1944. 456 p.

\section{d. Statistics}

A. H. Bowker \& H. P. Goode, Sampling Inspection by Variables, McGraw-Hill, New York-Toronto-London, 1952, $216 \mathrm{p}$. 
W. G. Cochran \& G. M. Cox, Experimental Designs. Wiley, New York, 1950. 454 p.

W. J. Dixon \& F. J. MASSEy, Introduction to Statistical Analysis. McGraw-Hill, New York-Toronto-London, $1951.370 \mathrm{p}$.

M. G. Kendall, The Advanced Theory of Statistics. Griffin, London, v. 1, 3rd edition, 1946, 457 p.; v. 2, 1946, 521 p.

A. WALD, Sequential Analysis. Wiley, New York, 1947. 212 p.

\section{e. Economics}

T. J. Koopmans, editor, Activity Analysis of Production and Allocation. Wiley, New York, 1951. 404 p. [RAND Report R-193.]

J. C. C. McKinsey, Introduction to the Theory of Games. McGraw-Hill, New YorkToronto-London, 1952. $371 \mathrm{p}$.

\section{REFERENCES ON PURE MATHEMATICS}

$$
\text { a. Logic }
$$

J. B. Rosser, Logic for Mathematicians. McGraw-Hill, New York, 1953. 530 p.

$$
\text { b. Algebra }
$$

G. Birkhoff \& S. MacLane, A Survey of Modern Algebra. Macmillan, New York, 1941. $450 \mathrm{p}$.

C. C. MacDuffee, The Theory of Matrices. (Reprint) Chelsea, New York, 1946. 110 p.

O. Perron, Die Lehre von den Kettenbrüchen. 2d edition, Teubner, Leipzig, 1929. 520 p.

S. Pertis, Theory of Matrices. Addison-Wesley, Cambridge, 1952. 237 p.

J. V. UsPensky, Theory of Equations. McGraw-Hill, New York, Toronto, and London, 1948. $353 \mathrm{p}$.

B. L. van DER WAERden (translated by F. BLuM), Modern Algebra. Ungar, New York, v. $1,1949,264$ p.; v. $2,1950,222$ p.

\section{c. Number theory}

G. H. Hardy \& E. M. Wright, An Introduction to the Theory of Numbers. Clarendon Press, Oxford, 1938. 403 p.

\section{d. Analysis in general}

E. Goursat (translated by E. R. Hedrick), A Course in Mathematical Analysis. Ginn, Boston, v. 1, 1904, 548 p. ; v. 2, part 1, 1916, 259 p.; v. 2, part 2, 1917, 300 p. [For v. 3 see next entry.]

E. Goursat, Cours d'Analyse Mathématique.v. 3, 5th edition, Gauthier-Villars, Paris, 1942. $702 \mathrm{p}$.

G. Pólya \& G. SzEgö, Aufgaben und Lehrsätze aus der Analysis. (Reprint) Dover, New York, 1945, v. 1, 342 p.; v. 2, 412 p.

\section{e. Calculus}

R. Courant (translated by E. J. McShane), Differential and Integral Calculus. Interscience, New York, v. 1, 1934, 616 p.; v. 2, 1936, 682 p.

G. H. HaRdy, $A$ Course of Pure Mathematics. 10th edition, Cambridge University Press, 1952. 509 p.

A. Ostrowski, Vorlesungen über Differential- und Integralrechnung. Birkhäuser, Basel, v. 1, 1945, 373 p.; v. 2, 1951, 482 p. [Chapter 6 of vol. 2 contains a rigorous treatment of numerical interpolation, integration, etc.]

G. E. F. Sherwood \& A. E. TAylor, Calculus. Revised edition, Prentice-Hall, New York, $1947.503 \mathrm{p}$. 


\section{f. Real variables}

L. M. Graves, The Theory of Functions of Real Variables. McGraw-Hill, New York, 1946. 300 p.

E. C. Titchmarsh, The Theory of Functions. 2d edition, Oxford University Press, London, 1939. $454 \mathrm{p}$.

\section{g. Complex variable; conformal maps}

E. F. Beckenbach, editor, Construction and Applications of Conformal Maps. National Bureau of Standards. Applied Mathematics Series, v. 18, Government Printing Office, 1952. 280 p. [Bibliography by W. SEIDEL on numerical methods.]

S. Bergman, The Kernel Function and Conformal Mapping. American Mathematical Society, New York, 1950. $161 \mathrm{p}$. $448 \mathrm{p}$.

E. T. Copson, Theory of Functions of a Complex Variable. Oxford University Press, 1935.

K. KNOPP (translated by F. BAGEMIHL), Theory of Functions. Dover, New York, v. 1, 1945,146 p.; v. $2,1947,150$ p.

Z. Nehari, Conformal Mapping. McGraw-Hill, New York-Toronto-London, 1952. 396 p.

h. Series, etc.

T. J. I'a. Bromwich, An Introduction to the Theory of Infinite Series. 2d edition, reprint, Macmillan, London, 1942. $535 \mathrm{p}$.

K. KNopp (translated by R. C. Young), Theory and Application of Infinite Series. Blackie, London, 1928. 571 p.

H. S. WALl, Analytical Theory of Continued Fractions. van Nostrand, New YorkToronto-London, 1948. 433 p.

\section{i. Fourier series; transforms}

W. Rogosinski (translated by H. Cohn and F. SteinhardT), Fourier Series. Chelsea, 1950. $176 \mathrm{p}$.

D. V. Widder, The Laplace Transform. Princeton University Press, 1941. 406 p.

A. Zygmund, Trigonometrical Series. Warsaw, 1935. $331 \mathrm{p}$.

\section{j. Polynomials and approximation}

N. I. AkHIEzer, Lektsii po Teorii Approksimatsii [Lectures on the Theory of Approximation]. Moscow-Leningrad, 1947. 323 p. [Being translated into German.]

D. JACKson, The Theory of Approximation. American Mathematical Society, New York, 1930. $178 \mathrm{p}$.

M. MARDEn, The Geometry of the Zeros of a Polynomial in a Complex Variable. American Mathematical Society, New York, 1949. 183 p.

G. SzEGö, Orthogonal Polynomials. American Mathematical Society, New York, 1939. $401 \mathrm{p}$.

J. L. WALSH, Interpolation and Approximation by Rational Functions in the Complex Domain. American Mathematical Society, New York, 1935, 382 p. $107 \mathrm{p}$.

J. M. Whitraker, Interpolatory Function Theory. Cambridge University Press, 1935.

\section{k. Special functions}

A. Erdelyi, W. Magnus, F. Oberhettinger, \& F. G. Tricomi, compilers, Higher Transcendental Functions. v. 1, McGraw-Hill, New York-Toronto-London, 1953. 302 p. [Based in part on notes left by HARRY BATEMAN.] 
E. W. Hobson, The Theory of Spherical and Ellipsoidal Harmonics. Cambridge University Press, $1931.500 \mathrm{p}$.

N. W. McLachlan, Theory and Application of Mathieu Functions. Clarendon Press, Oxford, 1947. $401 \mathrm{p}$.

F. Oberhettinger \& W. Magnus, Anwendungen der elliptischen Funktionen in Physik und Technik. Springer, Berlin-Göttingen-Heidelberg, 1949. 126 p.

J. B. Rosser, Theory and Application of $\int_{0}^{z} e^{-x^{2}} d x$ and $\int_{0}^{z} e^{-p^{2} y^{2}} d y \int_{0}^{y} e^{-x^{2}} d x$. Mapleton, Brooklyn, 1948. $192 \mathrm{p}$.

C. SNow, The Hypergeometric and Legendre Functions with Applications to Integral Equations of Potential Theory. National Bureau of Standards, Washington, 1942. $319 \mathrm{p}$.

M. J. O. Strutt, Lamésche, Mathieusche und verwandte Funktionen in Physik und Technik. (reprint) Edwards, Ann Arbor, 1944. 116 p.

J. TANNERy \& J. Molk, Eléments de la Théorie des Fonctions Elliptiques. GauthierVillars, Paris, v. 1, 1893, 246 p.; v. 2, 1896, 299 p.

G. N. Watson, A Treatise on the Theory of Bessel Functions. 2nd edition, Cambridge University Press, 1944. $804 \mathrm{p}$.

E. T. Whitraker \& G. N. Watson, A Course of Modern Analysis. American edition, Cambridge University Press, and Macmillan, New York, 1943. 608 p.

\section{Potential theory} $384 \mathrm{p}$.

O. D. KellogG, Foundations of Potential Theory. (Reprint) Ungar, New York, 1944.

\section{m. Differential equations}

H. Bateman, Partial Differential Equations of Mathematical Physics. (Reprint) Dover, New York, 1944. 522 p.

D. L. Bernstern, Existence Theorems in Partial Differential Equations. Princeton University Press, 1950. 228 p.

L. R. Ford, Differential Equations. McGraw-Hill, New York, 1933. 263 p.

P. FRANK \& R. vON MISES, Die Differential- und Integralgleichungen der Mechanik und Physik. 2d edition, 2 vs., reprint, Rosenberg, New York, 1943. $916+1106$ p.

E. L. INCE, Ordinary Differential Equations. (reprint) Dover, New York, c. 1945. 558 p.

E. KAMKE, Differentialgleichungen, Lösungsmethoden und Lösungen. 2 v. (Reprint), Edwards, Ann Arbor, 1945. $642+243$ p. $210 \mathrm{p}$.

S. Lefschetz, Lectures on Differential Equations. Princeton University Press, 1948.

N. Minorsky, Introduction to Non-linear Mechanics. Edwards, Ann Arbor, 1947. 464 p.

H. T. H. Piaggio, An Elementary Treatise on Differential Equations and their Applications. 7th edition, G. Bell, London, 1938. 256 p.

A. Sommerfeld (translated by E. G. Straus), Partial Differential Equations in Physics. Academic Press, New York, 1949. 335 p.

J. J. STOKER, Nonlinear Vibrations in Mechanical and Electrical Systems. Interscience, New York, 1950. 273 p.

J. D. Tamarkin \& W. Feller, Partial Differential Equations. Brown University, Providence, 1941. 268 p. [Mimeographed notes.]

A. G. Webster, Partial Differential Equations of Mathematical Physics. 2d edition, Stechert, New York, 1933. 440 p.

\section{n. Difference equations; integral equations}

H. BüCKNER, Die praktische Behandlung von Integral-Gleichungen. v. 1, Springer, Berlin, 1952. 127 p.

G. Kowalewski, Determinantentheorie. (Reprint) Chelsea, New York, 1948. 320 p. [Includes Fredholm determinants.] 

$558 \mathrm{p}$.

L. M. Minne-Thomson, The Calculus of Finite Differences. Macmillan, London, 1933.

N. E. NöRLund, Vorlesungen über Differenzenrechnung. (Reprint) Edwards, Ann Arbor, 1945. $551 \mathrm{p}$.

W. SCHMEIDLER, Integralgleichungen mit Anwendungen in Physik und Technik. I. Lineare Integralgleichungen. Akademische Verlagsgesellschaft, Leipzig, 1950. 611 p.

\section{o. Inequalities}

G. H. Hardy, J. E. Littlewood, \& G. Pólya, Inequalities. Cambridge University Press, 1934. $314 \mathrm{p}$.

T. Motzkin, Beiträge zur Theorie der linearen Ungleichungen. Azriel, Jerusalem, 1936. 71 p. [Translated into English by D. R. Fulkerson, Publication T-22, The RAND Corporation, Santa Monica, Calif., 7 March 1952, 86 p.]

p. Calculus of variations, etc. $296 \mathrm{p}$.

G. A. Buiss, Lectures on the Calculus of Variations. University of Chicago Press, 1946.

R. Courant \& D. Hilbert, Methoden der mathematischen Physik. 2d edition, 2 vols., reprint, Interscience, New York, 1943. $469+549 \mathrm{p}$.

\section{q. Probability}

H. Cramer, Mathematical Methods of Statistics. Princeton University Press, 1946. 575 p.

W. Feller, An Introduction to Probability Theory and its Applications. v. 1, Wiley, New York, 1950. $419 \mathrm{p}$.

J. V. Uspensky, Introduction to Mathematical Probability. McGraw-Hill, New York, 1937. $411 \mathrm{p}$.

$$
\text { r. Topology }
$$

M. H. A. Newman, Elements of the Topology of Plane Sets of Points. 2nd edition, Cambridge University Press, 1951. 214 p.

\section{s. Geometry}

P. J. Kelly \& H. Busemann, Projective Geometry and Projective Metrics. Academic Press, 1953. 332 p.

I. S. Sokolnikoff, Tensor Analysis, Theory and Applications. Wiley, 1951. 335 p.

D. J. Struik, Lectures on Classical Differential Geometry. Addison-Wesley, Cambridge, 1950. 221 p.

\section{NBSINA}

\section{George E. Forsythe}

This compilation was sponsored in part by the Office of Naval Research, USN. It represents the opinions of the author only, and not those of the National Bureau of Standards or the Office of Naval Research.

\section{On Finding the Characteristic Equation of a Square Matrix}

Various methods are known for finding explicitly the characteristic equation of a square matrix. ${ }^{1,2,3,4,5,6}$ Some of these make use of the CayleyHamilton theorem which states that every square matrix satisfies its own characteristic equation., ${ }^{3,45}$ In the present paper we describe among others, 